\title{
EFEITO DO TRATAMENTO TÉRMICO NA PROPORÇÃO DE FASES E NAS PROPRIEDADES MECÂNICAS DOS AÇOS INOXIDÁVEIS DUPLEX UNS S31803 E UNS S32304*
}

\section{Resumo}

Os aços inoxidáveis duplex (AID) possuem uma estrutura austeno-ferrítica de aproximadamente $50 \%$ de cada fase, tal característica confere aos AID propriedades mecânicas superiores aos aços inoxidáveis ferríticos e austeníticos e elevada resistência à corrosão. Esses aços possuem uma adição significativa de elementos de liga, que são os responsáveis pelo correto balanceamento das fases e asseguram as propriedades desejadas. Outro parâmetro importante para assegurar as propriedades desejadas é o processamento termomecânico. Sendo assim, este trabalho avaliou a influência de diferentes temperaturas de tratamento térmico de recozimento nas frações de fases primárias (ferrita e austenita) e nas propriedades mecânicas de dois AID (UNS S32304 e UNS S31803). Os tratamentos térmicos de recozimento foram realizados em cinco temperaturas diferentes com tempo de encharque fixo seguido de um resfriamento rápido. A quantificação das fases primárias foi realizada através de microscopia óptica (MO) e as propriedades mecânicas avaliadas em um ensaio de tração uniaxial. As frações de fases primárias medidas indicam que com o aumento da temperatura de recozimento há aumento na fração de ferrita e a temperatura onde a microestrutura é mais aproxima de $50 \%$ de ferrita e $50 \%$ de austenita foi em $1020^{\circ} \mathrm{C}$ em ambos os AID. Além disso, observou-se que com o aumento da temperatura e, consequentemente da fração de ferrita, houve uma redução no limite de resistência nos dois AID deste estudo.

Palavras-chave: Aços inoxidáveis; Tratamento térmico; Fração de fases; Propriedades mecânicas.

\section{EFFECT OF HEAT TREATMENT ON THE RATIO OF PHASES AND THE MECHANICAL PROPERTIES OF DUPLEX STAINLESS STEELS UNS S31803 AND UNS S32304}

\section{Abstract}

The duplex stainless steels (DSS) has an austenite-ferrite microstructure composed by about $50 \%$ of each phase, such characteristic gives to the DSS high corrosion resistance under strain. Those steels have significant addition of alloy elements, which are responsible for the appropriate phase balance and also ensure the desirable properties. Other important parameter for the correct phase balance is the thermomechanical processing. Therefore, this study evaluated the influence of different temperatures of heat treatment in the phase fraction (ferrite and austenite) and the mechanical properties of UNS S32304 and UNS S31803. The annealing were performed at five temperatures with fixed soaking time and followed by rapid cooling. The quantification of primary phases were performed through optical microscopy (OM) and the mechanical properties were evaluated in an uniaxial tensile test. The measurements of primary phase fraction indicate that with increasing annealing temperature there is an increase in ferrite fraction and the temperature where microstructure is more close to $50 \%$ ferrite and $50 \%$ austenite was around $1020^{\circ} \mathrm{C}$ in both DSS studied. Furthermore, it was observed that with increasing temperature and thus the ferrite fraction, there was a reduction in tensile strength in both DSS of this study.

Keywords: Duplex stainless steels; Heat treatment; Phase fraction; Mechanical properties.

1 Engenheira Metalurgista, Mestranda em Engenharia de Materiais, Universidade Federal de Ouro Preto (UFOP), Ouro Preto, MG, Brasil.

2 Eng. Mecânico, Doutor, Gerente Executivo, Centro de Pesquisa, Aperam South America, Timóteo, MG, Brasil.

3 Eng. Mecânico, Mestre, Pesquisador, Centro de Pesquisa, Aperam South America, Timóteo, MG, Brasil.

4 Físico, Doutor, Professor Adjunto, Depto. Engenharia Metalúrgica, UFOP, Ouro Preto, MG, Brasil. 


\section{INTRODUÇÃO}

Os aços inoxidáveis duplex (AID) vêm se apresentando como uma excelente alternativa para aplicações em que elevada resistência à corrosão e alta resistência mecânica são requeridas [1]. As principias aplicações dos AID estão concentradas no setor de óleo e gás. Assim, é fundamental estudos destes aços para ampliar seu uso neste setor e expandir à outros segmentos industriais.

A microestrutura dos AID é caracterizada por apresentar duas fases primárias: austenita e ferrita em proporções aproximadamente iguais [2,3]. Os AID são uma família de tipos de aços, que variam em desempenho frente à corrosão, dependendo do teor de elementos de liga. Os AID mais modernos podem ser divididos em cinco grupos [3]:

- Lean Duplex, como o 2304, que não contém adição deliberada de molibdênio;

- Duplex padrão, como o 2205, que são os mais fabricados;

- Duplex $25 \mathrm{Cr}$, que possuem uma boa resistência à corrosão por pites;

- Super Duplex, com elevada resistência à corrosão por pites;

- Hiper Duplex, que são altamente ligados.

A maior parte dos AID se solidifica como ferrita $(\delta)$ e a transformação em austenita $(\gamma)$ ocorre no resfriamento [4]. A solidificação se inicia abaixo de $1450^{\circ} \mathrm{C}$ totalmente como ferrita e continuando o resfriamento, a austenita se forma em temperaturas por volta de $1000^{\circ} \mathrm{C}$, dependendo da composição da liga [3]. A precipitação de austenita iniciará na interface $\delta / \delta+\gamma$ e a quantidade de austenita precipitada dependerá da taxa de resfriamento. Quanto menor essa taxa, mais austenita será formada [5].

Pequenas mudanças na composição podem ter um efeito significativo sobre a fração volumétrica das fases. O balanço de fases desejado em um AID é atingido principalmente ajustando os teores de cromo, molibdênio, níquel e nitrogênio, e em seguida controlando o processamento termomecânico [3].

Tendo em vista a importância do processamento termomecânico para se alcançar as propriedades desejáveis nos AID, este estudo avaliou o efeito de diferentes temperaturas de tratamentos térmico de recozimento na fração de fases primárias e nas propriedades mecânicas dos AID UNS S31803 e UNS S2304.

\section{MATERIAIS E MÉTODOS}

Foram utilizadas amostras dos AID UNS S31803 e UNS S32304 planos, produzidas industrialmente na Empresa Aperam South America. Estas amostras foram retiradas após a etapa de laminação a quente e recozimento industrial e têm espessura de 4,00mm. A Tabela 1 apresenta a faixa de composição química dos referidos aços.

Tabela 1. Faixa de composição química (\% em peso) dos AID UNS S32304 e UNS S31803

\begin{tabular}{lcccccccccc}
\hline Aço & $\mathbf{C}$ & $\mathbf{C r}$ & $\mathbf{N i}$ & $\mathbf{M o}$ & $\mathbf{N}$ & $\mathbf{M n}$ & $\mathbf{C u}$ & $\mathbf{S i}$ & $\mathbf{S}$ & $\mathbf{P}$ \\
\hline UNS S32304 & 0,01 & 22,87 & 4,20 & 0,28 & 0,12 & 1,45 & 0,45 & 0,20 & 0,0004 & 0,02 \\
\hline UNS S31803 & 0,03 & 22,44 & 5.31 & 2,63 & 0,15 & 1,81 & 0,24 & 0,38 & 0,0005 & 0,03 \\
\hline
\end{tabular}

As amostras dos AID foram laminadas a frio para redução de espessura de $4,00 \mathrm{~mm}$ para $1,85 \mathrm{~mm}$. Essas laminações foram realizadas em laboratório usando um laminador piloto, modelo 4.085, do fabricante FENN.

Após etapa de laminação a frio as amostras foram destinadas ao processo de tratamento térmico de recozimento. Esses recozimentos foram realizados em um forno estacionário tipo Mufla, modelo Combustol. Foram feitas cinco temperaturas 
diferentes para os recozimentos: $990^{\circ} \mathrm{C}, 1020^{\circ} \mathrm{C}, 1050^{\circ} \mathrm{C}, 1080^{\circ} \mathrm{C}$ e $1110^{\circ} \mathrm{C}$ e para todas as temperaturas foi utilizado encharque de 25 segundos, seguido por resfriamento rápido em ambiente contendo água e gelo, com o objetivo de evitar transformações de fase durante o resfriamento.

As amostras depois de recozidas foram preparadas para análise metalográfica quantitativa e para serem testadas em um ensaio de tração uniaxial.

A medição das fases primárias foi feita em um microscópio óptico (MO) LEICA DM RM equipado com um software analisador de imagem. Foram medidos 20 campos em cada amostra, escolhidos aleatoriamente.

Os ensaios de tração uniaxial foram realizados para determinar a influência dos tratamentos térmicos nas propriedades mecânicas. Para esse ensaio foram confeccionados três corpos de prova de secção retangular conforme a norma ASTM E8M com $300 \mathrm{~mm}$ de comprimento e $50 \mathrm{~mm}$ de largura. Os corpos de prova foram ensaiados na direção longitudinal usando um equipamento INSTRON modelo 5583, equipada com célula de carga de 15 toneladas com um vídeo extensômetro infravermelho e software Bluehill.

\section{RESULTADOS E DISCUSSÃO}

As micrografias apresentadas na Fig 1 e Fig 2 mostram as microestruturas típicas dos AID UNS S32304 e UNS S31803, respectivamente, nas diferentes temperaturas de recozimento. A fase escura representa a ferrita e a clara a austenita. Destacamse ainda, o alongamento dessas fases na direção de laminação em ambos os AID.

(a)

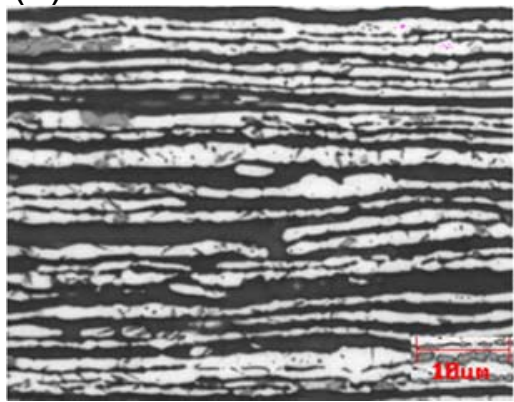

(d) (b)

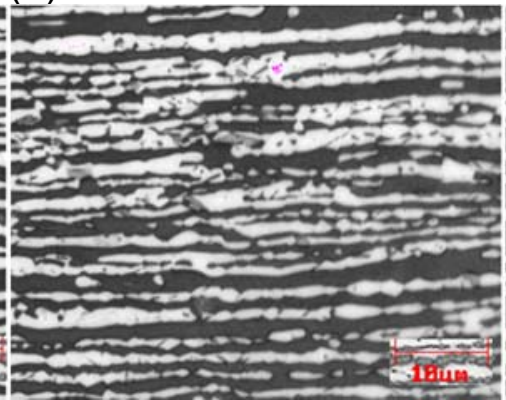

(e) (c)
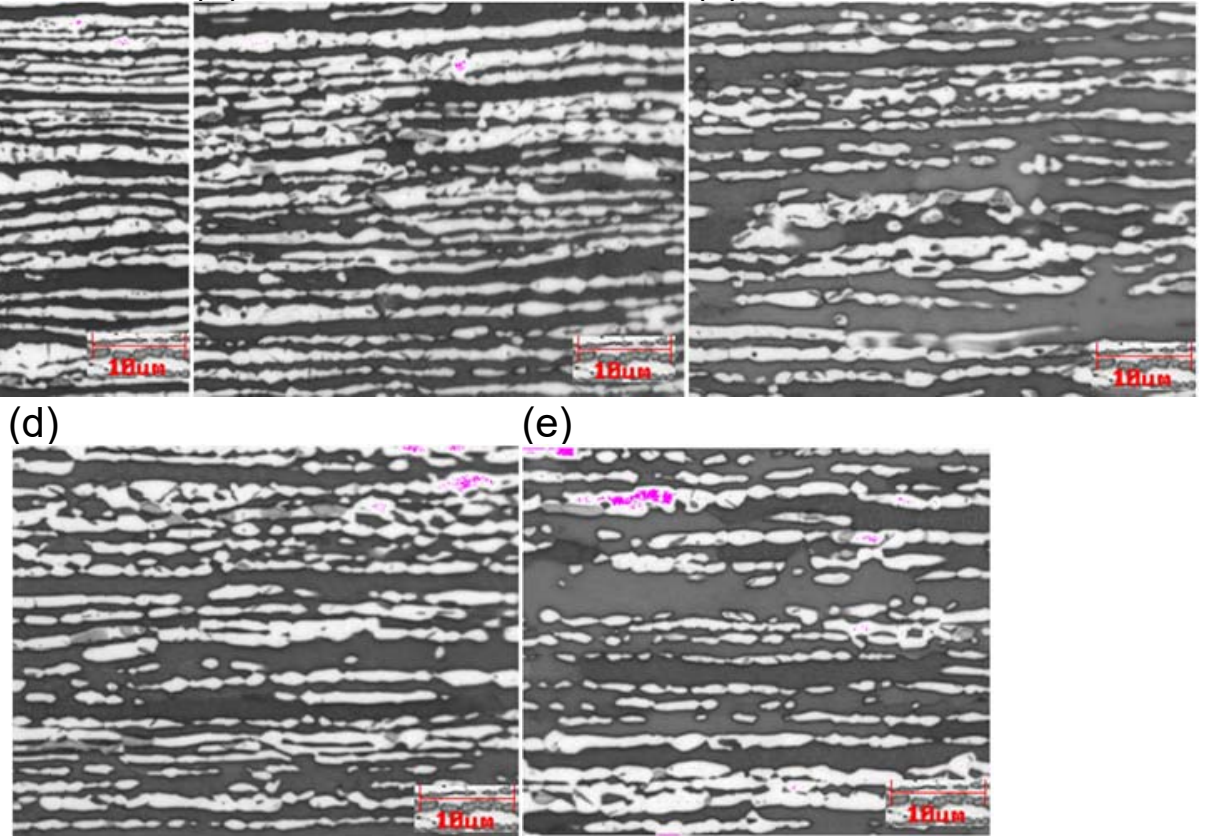

Figura 1. Microestrutura do AID UNS S32304: (a) $990^{\circ} \mathrm{C}$; (b) $1020^{\circ} \mathrm{C}$; (c) $1050^{\circ} \mathrm{C}$; (d) $1080^{\circ} \mathrm{C}$ e (e) $1110^{\circ} \mathrm{C}$ 
(a)

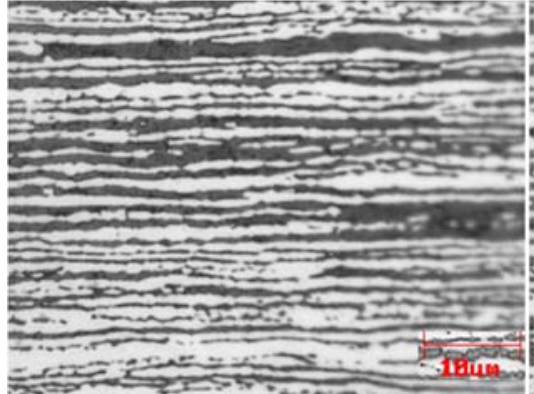

(d) (b)

(c)

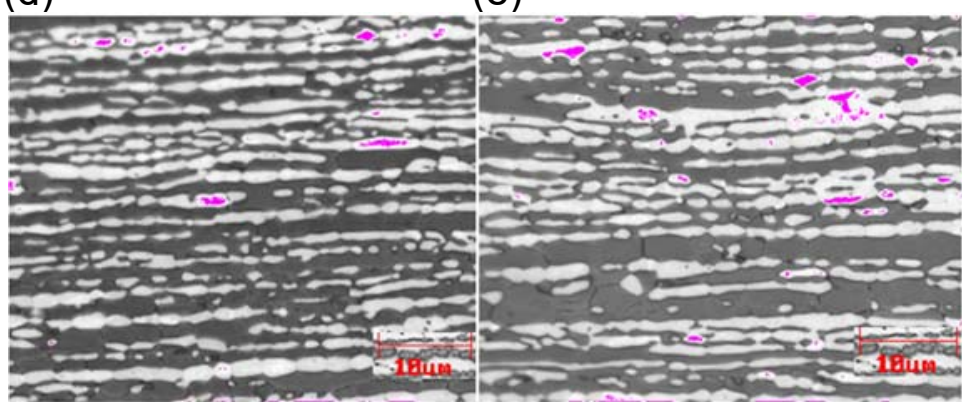

Figura 2. Microestrutura do AID UNS S31803: (a) $990^{\circ} \mathrm{C}$; (b) $1020^{\circ} \mathrm{C}$; (c) $1050^{\circ} \mathrm{C}$; (d) $1080^{\circ} \mathrm{C}$ e (e) $1110^{\circ} \mathrm{C}$.

As Figs 3 e 4 apresentam a evolução da transformação das fases primárias ferrita e austenita com o aumento da temperatura de recozimento para os AID estudados.

De acordo com os resultados obtidos, a temperatura em que ambos os aços atingem aproximadamente $50 \%$ de ferrita e $50 \%$ de austenita foi $1020^{\circ} \mathrm{C}$.

Com o aumento da temperatura de recozimento a fração de ferrita aumentou chegando a $64 \%$ no aço UNS S32304 e $63 \%$ no aço UNS S31803 à $1110^{\circ} \mathrm{C}$. Esse resultado é compatível com os diagramas de fases, pois em elevadas temperaturas a fase mais presente é a ferrita [3].

Outra observação que pode ser feita é que a $990^{\circ} \mathrm{C}$ o aço UNS S32304 possui $54 \%$ de ferrita e $46 \%$ de austenita, enquanto o UNS S31803 possui $48 \%$ de ferrita e $52 \%$ de austenita. Essa menor fração de ferrita no UNS S31803 pode estar relacionada a fenômenos de precipitação de fases secundárias e compostos intermetálicos (carbonetos, nitretos, sigma, chi, dentre outros) [6]. 


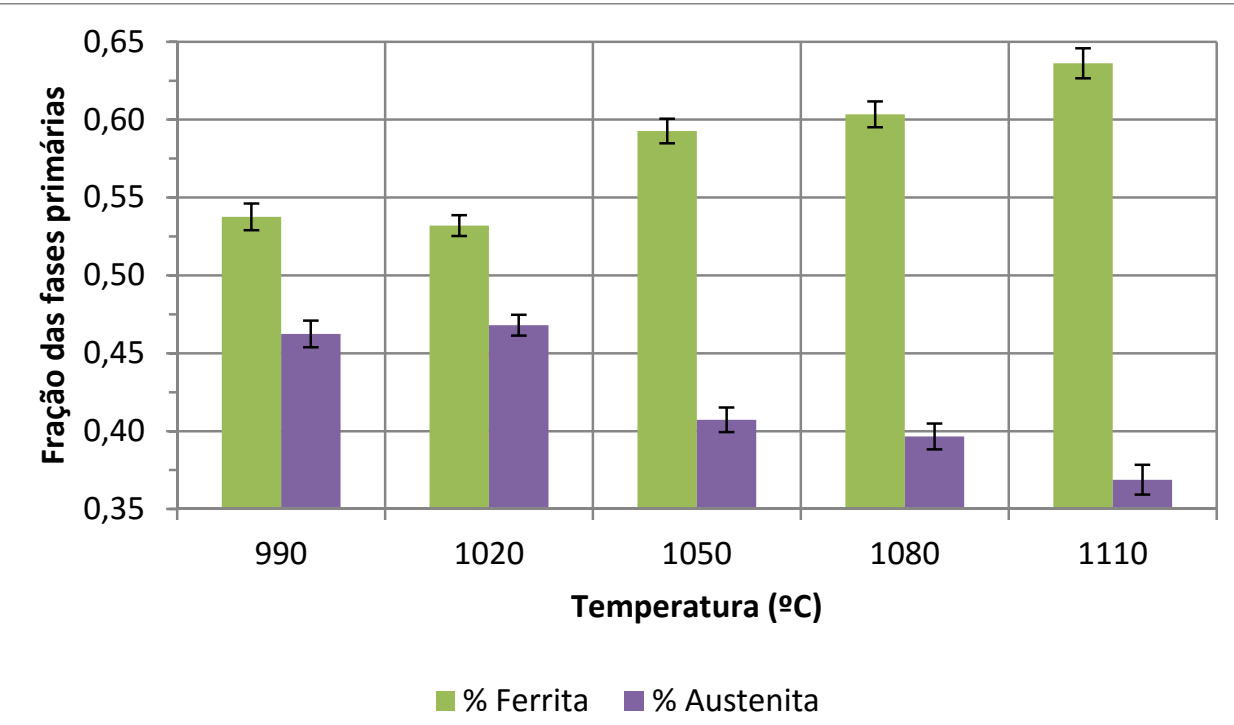

Figura 3. Fração das fases primárias em diferentes temperaturas para o AID UNS S32304.

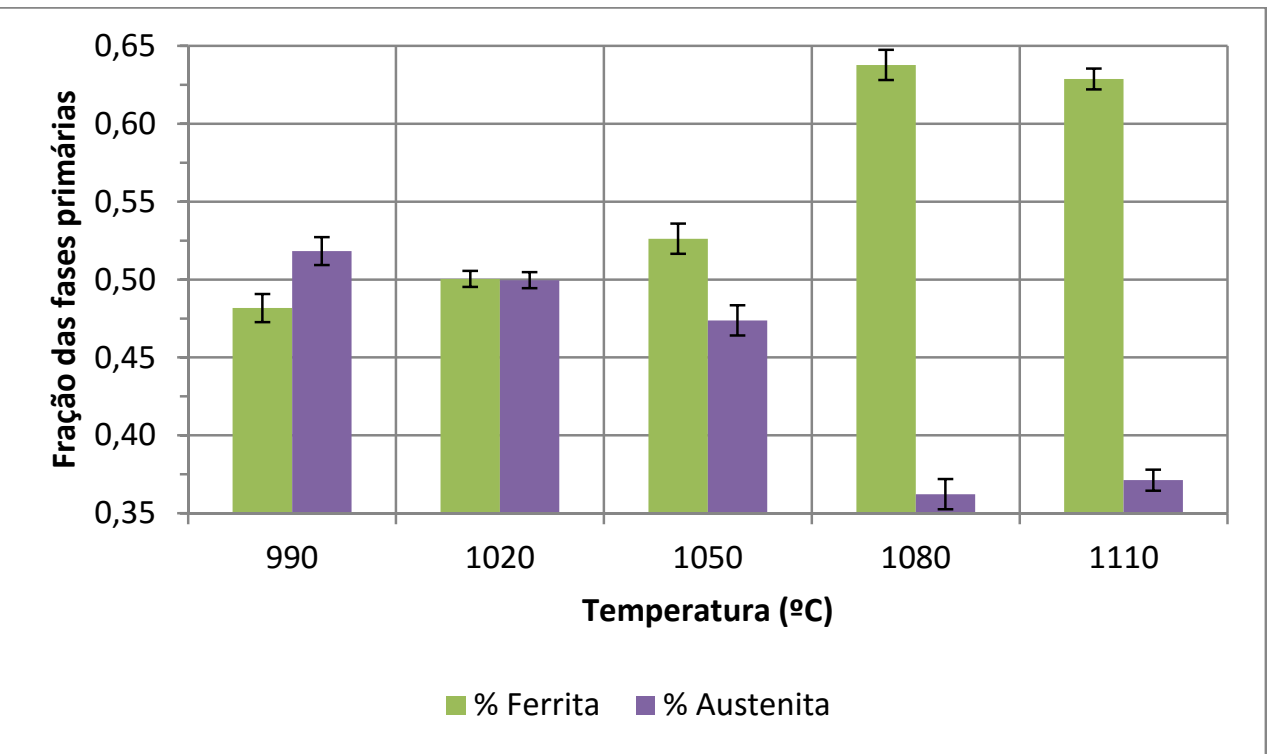

Figura 4. Fração das fases primárias em diferentes temperaturas para o AID UNS S31803.

As Figs 5 e 6 apresentam a relação entre os valores de fração das fases primárias nas diferentes temperaturas de recozimento e sua relação com o limite de resistência (LR). 


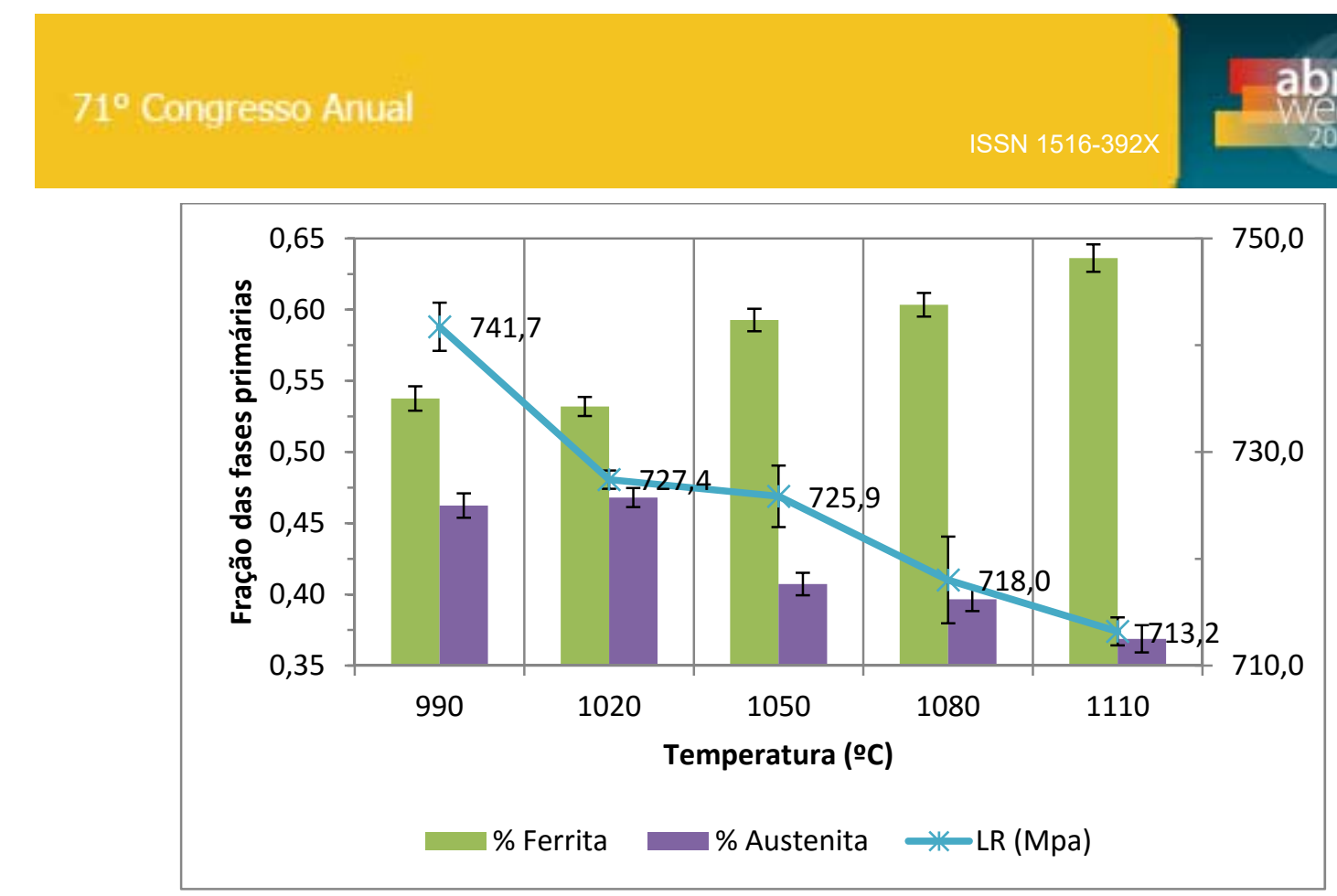

Figura 5. Relação entre fração das fases primárias e os valores de limite de resistência medidos nas diferentes temperaturas de recozimento no AID UNS S32304.

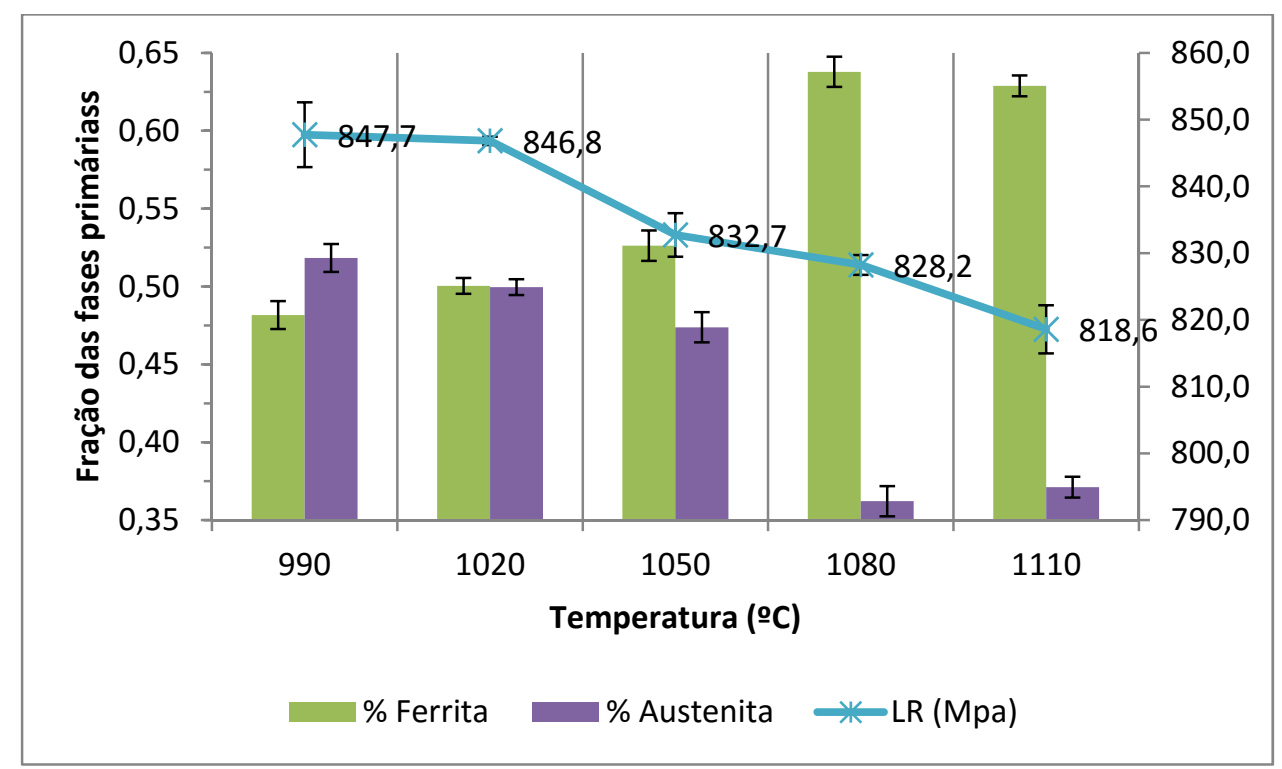

Figura 6. Relação entre fração das fases primárias e os valores de limite de resistência medidos nas diferentes temperaturas de recozimento no AID UNS S31803.

Os resultados do ensaio de tração uniaxial mostram que com o aumento da temperatura, e consequentemente aumento na fração da fase ferrita, há uma redução no limite de resistência para os dois AID. Isso provavelmente está relacionado com o comportamento mecânico da austenita, que possui maior expoente de encruamento que da ferrita [7]. Sendo assim, frações próximas a $50 \%$ de austenita conferem melhor resistência mecânica aos AID.

É possível observar também que o limite de resistência é maior no AID UNS S31803 do que no UNS S32304 em todas as temperaturas de recozimento. A principal razão é que no UNS S31803 há maior adição de molibdênio e nitrogênio. Ambos os elementos causam distorção na rede cristalina que dificulta a movimentação das discordâncias e promove o endurecimento por solução sólida. O molibdênio causa distorção na rede devido ao fato de seu raio atômico ser maior que o raio do solvente, e o nitrogênio por possuir átomos menores que os do solvente, que ocupam as posições intersticiais [8]. 
Medidas de limite de escoamento (LE), alongamentos uniforme (AU) e total (AT) e dureza também foram realizadas, porém, não foi encontrada uma correlação entre estes parâmetros e as medições de fração das fases nas temperaturas de recozimento usadas neste estudo.

\section{CONCLUSÃO}

- Com o aumento da temperatura de recozimento houve um aumento na fração da fase ferrita e consequente redução de austenita;

- Tanto para o UNS S32304 quanto para o UNS S31803 a temperatura de recozimento onde a estrutura mais se aproximou de $50 \%$ de ferrita e $50 \%$ de austenita foi $1020^{\circ} \mathrm{C}$;

- O aumento na fração da fase ferrita ocasionou diminuição no valor do limite de resistência nos dois aços, provavelmente gerado pela redução de austenita, que possui maior expoente de encruamento;

- Os valores de limite de resistência foram maiores para o aço UNS S31803 do que para o UNS S32304, em todas as temperaturas de recozimento, devido ao fenômeno de endurecimento por solução sólida promovido pela maior quantidade de molibdênio e nitrogênio.

\section{Agradecimentos}

A Aperam South America pelo apoio na realização deste projeto.

\section{REFERÊNCIAS}

1 Costa e Silva, A.L.V.; Mei, P.R. Aços e ligas especiais. $3^{\circ}$ Edição. São Paulo: Blucher, 2010.

2 Solomon, H. D.; Devine Jr, T. M. Duplex stainless steel - a tale of two phases. In: Duplex Stainless Steel. Proceedings Metals Park, Ohio: ASM, 1982, p. 693-756.

3 Nilsson, J. O. Super duplex stainless steels. Materials Science and Technology. Volume 8, 1992, p. 685-700.

4 International Molybdenum Association (IMOA). Practical Guidelines for the Fabrication of Duplex Stainless Steel. $2^{\mathrm{a}}$ Edição, London - UK, 2009.

5 Charles, J., and P. Chemelle. The history of duplex developments, nowadays DSS properties and duplex market future trends. World Iron \& Steel 6 (2011): 002.

6 Costa e Silva, A. L., Avillez, R. R. Avaliação da cinética de precipitação de compostos intermetálicos em aços inoxidáveis duplex através de modelamento matemático da difusão. $64^{\circ}$ Congresso Anual da ABM, 2009, Belo Horizonte, Brasil.

7 Shackelford, J. F. Introdução à ciência dos materiais para engenheiros. São Paulo: Pearson Prentice Hall, 2008, p. 124-125.

8 Dieter, G. E. Metalurgia Mecânica. $2^{\mathrm{a}}$ Edição. Rio de Janeiro: Guanabara Koogan S.A., 1981. 